\title{
Daidzein inhibits choriocarcinoma proliferation by arresting cell cycle at G1 phase through suppressing ERK pathway in vitro and in vivo
}

\author{
WEI ZHENG ${ }^{1}$, RONG SUN ${ }^{2}$, LEI YANG $^{1}$, XIANLING ZENG $^{1}$, YAN XUE $^{1}$ and RUIFANG AN ${ }^{1}$ \\ ${ }^{1}$ Department of Gynecology and Obstetrics, The First Affiliated Hospital of Xi'an Jiaotong University, Xi'an, \\ Shaanxi 710061; ${ }^{2}$ Obstetrics and Gynecology Hospital, Fudan University, Shanghai 200012, P.R. China
}

Received March 23, 2017; Accepted August 17, 2017

DOI: $10.3892 /$ or.2017.5928

\begin{abstract}
Choriocarcinoma is a highly malignant tumor arising from abnormal gestational trophoblast proliferation. Although chemotherapy has dramatically improved the prognosis, there are still some patients who become drug-resistant or relapse. Daidzein has garnered interest in its antitumor activity especially in proliferation inhibition. However, few reports exist on daidzein effect in growth of choriocarcinoma. Therefore, in this study, we performed in vitro and in vivo experiment in JAR and JEG-3 to investigate the effect of daidzein in proliferation of choriocarcinoma. Daidzein inhibited cell growth in a time- and dose-dependent way. Cell cycle was arrested at G1 phase and expression of cyclin D1, c-myc, PCNA was reduced while p21 was upregulated during daidzein treatment. At the same time, the expression of p-ERK was downregulated and translocation into nuclear afterwards was also inhibited. Moreover, ERK agonist ceramide C6 abolished daidzein's effects on cell proliferation. Besides, in vivo experiment also showed daidzein's anti-proliferation function as xenografts growth was inhibited and expressions of c-myc, PCNA and p-ERK were suppressed. In conclusion, results in our study demonstrate daidzein can inhibit choriocarcinoma cell proliferation in vitro and in vivo; underlying mechanism behind the inhibitory effects may probably be suppressing ERK pathway and afterwards arresting cell cycle at G1 phase.
\end{abstract}

\section{Introduction}

Gestational trophoblastic disease is a spectrum of trophoblast abnormal proliferation (1), among which choriocarcinoma is the

\footnotetext{
Correspondence to: Professor Ruifang An or Dr Yan Xue, Department of Gynecology and Obstetrics, The First Affiliated Hospital of Xi'an Jiaotong University, 277 Yanta Western Road, Xi'an, Shaanxi 710061, P.R. China

E-mail: ruifangan@163.com

E-mail: xueyan325@126.com
}

Key words: daidzein, choriocarcinoma, proliferation, G1 phase, ERK, in vitro, in vivo most malignant one tending to spread to lung, liver and/or brain. Though most choriocarcinoma occurs after a complete mole, it may also be secondary to any normal or abnormal pregnancy, including partial mole, term pregnancy, induced/spontaneous abortion, premature delivery and stillbirth (2). However, $25 \%$ of gestational trophoblastic tumors will be resistant to, or will relapse after initial chemotherapy $(3,4)$. Also, in development of gestational trophoblastic disease, excess proliferation is an important clue and is related with outcome of treatment $(5,6)$. Therefore, we are wondering if there is an alternative medicine which may inhibit trophoblastic cell proliferation and improve the curative rate of choriocarcinoma.

Cell proliferation is regulated by positive and negative cell cycle regulatory factors. Cyclin-dependent kinases (CDKs) are the key factors forming CDKs/cyclins complexes (such as CDK4/cyclin D1), while CDK inhibitors (CKIs), such as p21 and $\mathrm{p} 15$, are negative regulators acting as the brakes of the cell cycle transition by obstructing the formation of CDK/cyclin complexes $(7,8)$. Activation of $\mathrm{CDK} /$ cyclins is required for cell cycle progression through the G1/S checkpoint. Proliferation nuclear antigen (PCNA), associated with DNA synthesis, functions in the cell cycle progression from early $\mathrm{G} 1$ to $\mathrm{S}$ phase, and its function is inhibited when p21 binds to it (9). c-myc positively regulates the expression and/or activity of cyclins (D1, D2, E and A), CDK2/CDK4, and additionally suppresses CDKIs such as p15, p21 and p27 (10). Therefore, these proteins are often used to indicate the interference on the cell cycle. Also, arresting cell cycle in G1 phase or G2/M phase is one of the mechanisms used by anticancer medicines.

Cellular proliferation is strongly regulated by mitogenactivated protein kinase (MAPK), including extracellular signal-regulated kinase 1/2 (ERK1/2), c-Jun N-terminal kinase (JNK), and p38 MAPK, in a variety of cell types. The ERK1/2 signaling pathway, activated by phosphorylation and subsequent nuclear translocation, has been implicated as a regulator of cell cycle arrest $(11,12)$. Also, ERK signal pathway is involved in trophoblast cell proliferation and invasion $(13,14)$.

Recently, the antitumor effects of ingredients from diet have been paid attention to because of their accessibility and security. Daidzein belongs to the isoflavones family, one of the most commonly ingested and most intensely studied type of phytoestrogen, often found in nuts, fruits, soybeans, and 
soy-based products (15). Previously, daidzein garnered interest in its antitumor activity especially in proliferation inhibition and apoptosis induction. Daidzein induces apoptosis in prostate cancer cells (LnCap and PC3) (16), and its metabolite inhibits prostate cancer growth in vitro and in vivo (17). In breast cancer, daidzein shows antiproliferation activity, causing cell cycle arrest at the G1 and G2/M phases and its metabolite enhance tamoxifen's caspase-mediate apoptosis induction $(18,19)$. Daidzein also plays its antitumor role in colon cancer, arresting cell cycle at G0/G1 phase (20), as well as other cancers such as cervical cancer, melanoma, hepatic cancer and gastric carcinoma effecting proliferation and/or apoptosis (21-24). As to trophoblast cells, Jeschke et al found a significant decrease of hCG production in daidzein-treated trophoblast cells in a concentration-dependent way (25). However, scarce information on daidzein effect in growth of choriocarcinoma is available.

Moreover, daidzein has a close relationship with ERK. Soy isoflavones decreases the expression causing cell cycle arrest and upregulation of p21 in colon adenocarcinoma (26). Daidzein suppresses phosphorylation levels of ERK when exerting antitumor activity against bladder cancer cells (27). Also, its metabolites are able to inhibit the activation of ERK $(28,29)$.

We hypothesized that daidzein can inhibit choriocarcinoma cell proliferation by arresting the cell cycle, and this may due to the suppression of expression of ERK pathway.

\section{Materials and methods}

Cell culture. Choriocarcinoma cell lines JAR and JEG-3 obtained from American Type Culture Collection (Manassas, VA, USA) were cultured in DMEM medium with $10 \%$ fetal bovine serum at $37^{\circ} \mathrm{C}$ with $5 \% \mathrm{CO}_{2}$. ERK was activated by $10 \mu \mathrm{M}$ concentration of ceramide C6 (C-C6; Santa Cruz Biotechnology, Santa Cruz, CA, USA), added $24 \mathrm{~h}$ after daidzein treatment for another $24 \mathrm{~h}$.

MTT assay. Growth rates of cells were measured by 3(4,5-dimethylthiazol-2-yl)-2,5-diphenyltetrazolium bromide (MTT) (Sigma, St. Louis, MO, USA) assay. The absorbance was measured at $490 \mathrm{~nm}$ using a universal microplate reader (Model ELx800; BioTek Instruments, Inc., Winooski, VT, USA).

Colony formation assay. JAR and JEG-3 cells were seeded onto 6-well plates at a density of 1,000 cells per well. After 14 days of culture, cells were washed with PBS, fixed with $4 \%$ paraformaldehyde, and subsequently stained with $0.1 \%$ crystal violet solutions.

Cell cycle analysis. Cells with 60-80\% confluence were trypsinized, washed with cold PBS, resuspended with cold $70 \%$ ethanol then stored at $-20^{\circ} \mathrm{C}$ overnight. Before subjected to flow cytometry analysis, ethanol was removed and pellet cells were washed with cold PBS twice, cells were resuspended in PBS with $0.5 \mu \mathrm{g} / \mathrm{ml}$ RNase and $50 \mu \mathrm{g} / \mathrm{ml}$ propidium iodide and incubated at room temperature in the dark for $30 \mathrm{~min}$. Then cell samples were analyzed in a FACSCalibur flow cytometer (Becton-Dickinson, San Jose, CA, USA) and CellQuest software.
Western blot analysis. Cells were washed once with cold PBS and lysed in RIPA buffer (50 mM Tris pH 8.0, $150 \mathrm{mM}$ $\mathrm{NaCl}, 0.1 \%$ SDS, $1 \%$ NP-40, and $0.5 \%$ sodium deoxycholate) containing protease inhibitors. Approximately $20 \mu \mathrm{g}$ of protein was separated with $8-12 \%$ SDS-PAGE gel and blotted onto nitrocellulose membranes. Then membranes were blocked with $5 \%$ skim milk at room temperature for $1 \mathrm{~h}$ and then incubated with primary antibodies against $\beta$-actin (Abcam, Cambridge, UK), c-myc (Abcam), PCNA (Santa Cruz), cyclin D1 (Santa Cruz), p21 (Cell Signaling Technology, MA, USA), ERK (Abcam) and p-ERK (Abcam) at $4^{\circ} \mathrm{C}$ overnight, followed by TBST wash and 1-h incubation with HRP-conjugated secondary antibodies at room temperature. Protein bands were visualized by a Molecular Imager ChemiDoc XRS System (Bio-Rad Laboratories, Hercules, CA, USA).

Immunofluorescence microscopy. Cells were fixed in $4 \%$ paraformaldehyde in PBS for $15 \mathrm{~min}$, and then permeabilized with $0.1 \%$ Triton X-100 for $15 \mathrm{~min}$ and blocked in $1 \%$ BSA for $1 \mathrm{~h}$. Cells were sequentially incubated with primary antibody (p-ERK; $1: 100$ in $1 \% \mathrm{BSA}$ ) at $4^{\circ} \mathrm{C}$ overnight, followed by Alexa Fluor 488 second antibody (1:150 in 1\% BSA) for $1 \mathrm{~h}$ and DAPI for $5 \mathrm{~min}$. Slides were analyzed and photographed by Olympus BX51 Microscope (Olympus, Tokyo, Japan).

Xenograft tumor model. Fifteen mice were randomly divided into three groups depending on different gavage components: negative control group (edible oil only), low dose group (10 $\mathrm{mg} / \mathrm{kg}$ body weight/day of daidzein dissolved in oil) and high dose group $(20 \mathrm{mg} / \mathrm{kg}$ body weight/day of daidzein dissolved in oil). For tumorigenesis assay in nude mice, $5 \times 10^{6}$ JEG-3 (considering JEG-3 more easily formed subcutaneous xenografts than JAR when performing pre-experiment) were injected subcutaneously into left sides of the flank region after 1 week daily gavage feeding. Three weeks after subcutaneous injection with JEG-3 (4 weeks after daily gavage with/without different concentration of daidzein), the mice were sacrificed, then xenograft tumors were harvested, weighed and fixed with $4 \%$ paraformaldehyde. Animal care and protocols were in accordance with the guidelines of the Institutional Animal Care and Use Committee of Xi'an Jiaotong University.

Immunohistochemistry. Tumor sections of nude mouse xenografts were studied by immunohistochemistry (IHC) assay using EnVision ${ }^{\mathrm{TM}}$ System (Dako, Carpinteria, CA, USA). Primary antibodies used in IHC were c-myc (Abcam), PCNA (Santa Cruz), p-ERK (Abcam). Staining signals were photographed using an Olympus BX51 microscope (Olympus). Average intensity score of the positive cells $(0$, none; 1 , weak; 2 , intermediate; 3 , strong) and percentage score of stained cells $(1,0-25 \% ; 2,25-50 \% ; 3,50-75 \% ; 4,75-100 \%)$ were multiplied to get the total staining score, ranging from 0 to 12 .

Statistical analysis. All experiments were repeated at least three times. Statistical analysis was carried out using the SPSS 19.0 statistical software package (SPSS, Inc., Chicago, IL, USA) was used to analyze differences between two groups (Student's t-test) and p-values $<0.05$ were regarded as statistically significant. 
A

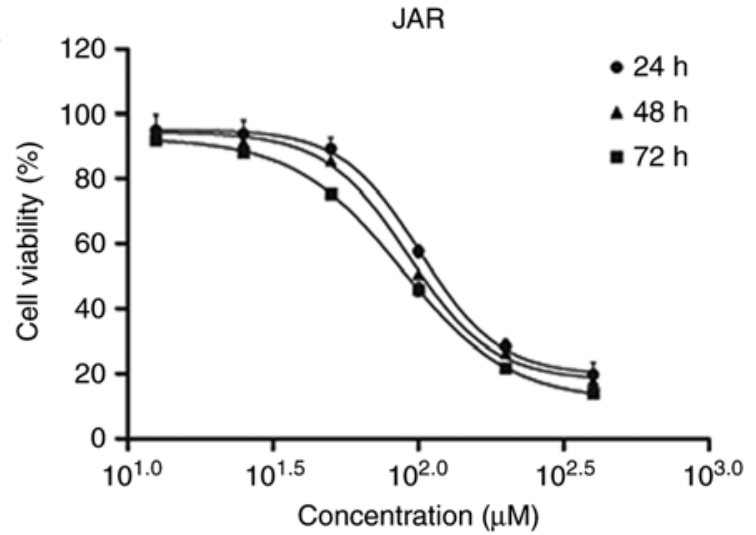

B

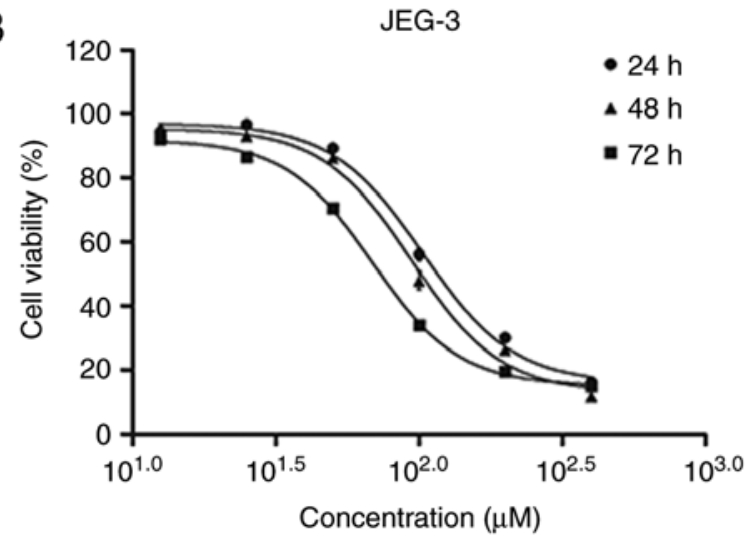

Figure 1. The influence of daidzein on choriocarcinoma cell growth. (A) Cell viability of JAR treated with different concentrations of daidzein after 24,48 and $72 \mathrm{~h}$ determined by MTT assay. (B) Cell viability of JEG-3 treated with different concentrations of daidzein after 24, 48 and $72 \mathrm{~h}$. The results are expressed as a percentage of viable cells compared with control cells.

A JAR

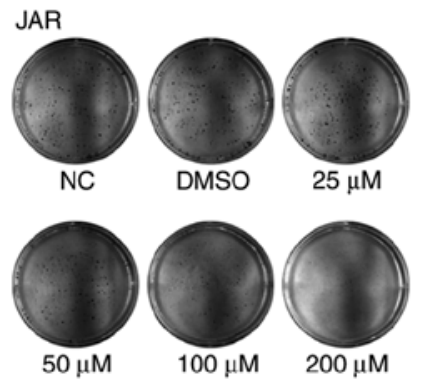

B

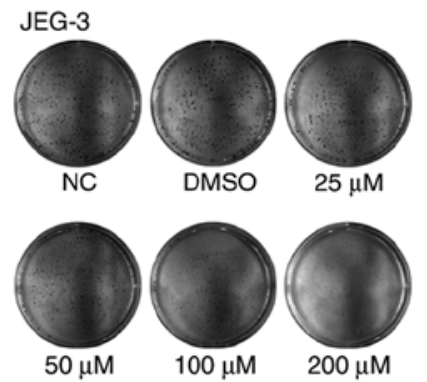

C

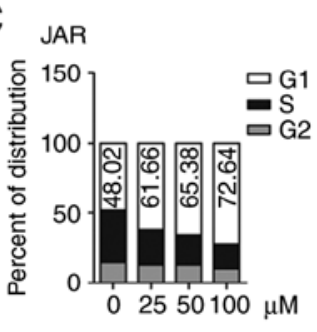

D

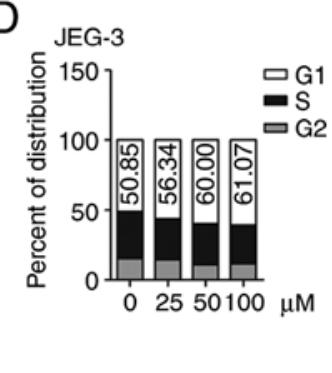

$\mathrm{E}$

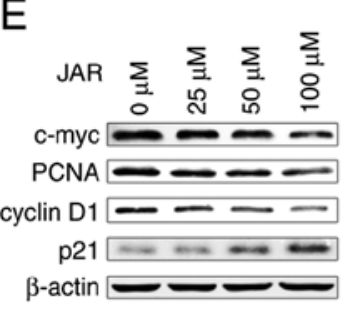

F

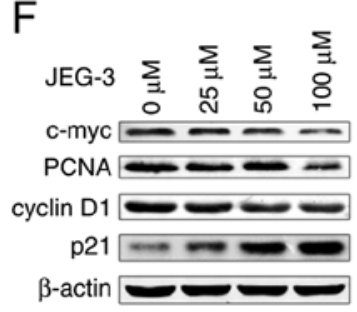

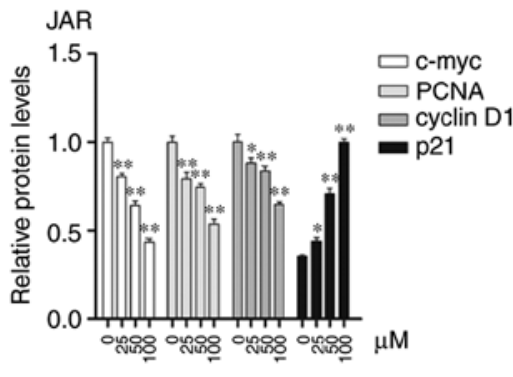

JEG-3

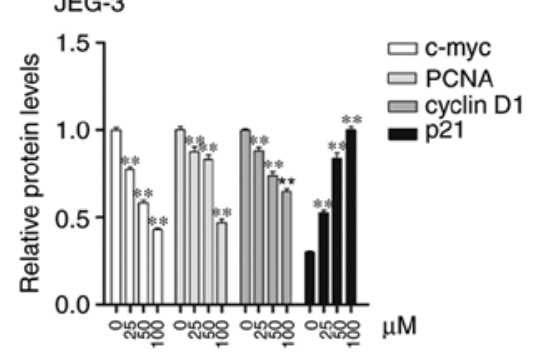

Figure 2. The effect of different concentration of daidzein on choriocarcinoma proliferation. (A) Colony formation assay of JAR. (B) Colony formation assay of JEG-3. (C) Cell cycle distribution of JAR detected by flow cytometry. (D) Cell cycle distribution of JEG-3. (E) Western blotting and the quantification of proliferation associating markers in JAR. $\beta$-actin was used as internal control. (F) Western blotting and the quantification of proliferation associating markers in JEG-3. Compared with $0 \mu \mathrm{M}, \stackrel{*}{\mathrm{p}}<0.05,{ }^{* *} \mathrm{p}<0.01$.

\section{Results}

Daidzein reduces cell viability in choriocarcinoma cell lines. Using MTT assay, we investigated the viability inhibition effect of daidzein in CC cell lines JAR and JEG-3. Both cell lines were treated with daidzein at concentration of $12.5,25$, $50,100,200$ and $400 \mu \mathrm{M}$. The results showed that daidzein had a concentration- and time-dependent effect on JAR cell viability. With the increasing of concentration or time of treatment with daidzein, the viability decreased. Also, the $\mathrm{IC}_{50}$ at 24,48 and $72 \mathrm{~h}$ were $101.60,92.56$ and $87.73 \mu \mathrm{M}$, respectively (Fig. 1A). Similar effect were seen in JEG-3, while the $\mathrm{IC}_{50}$ was 103.10, 94.14 and $68.82 \mu \mathrm{M}$, respectively (Fig. 1B).

Daidzein treatment inhibits proliferation of choriocarcinoma cells. In order to determine whether the cell viability reduction is due to proliferation inhibition, we performed colony formation assay on both cell types with daidzein treatment. The colony-forming capacity of JAR and JEG-3 decreased as the concentration of daidzein increased. While $25 \mu \mathrm{M}$ daidzein seemed not to change much, treatment with 50 and $100 \mu \mathrm{M}$ daidzein markedly suppressed proliferation. However, when the concentration reached $200 \mu \mathrm{M}$, colonies hardly formed (Fig. 2A and B).

Cell cycle is arrested in G1 phase when treated with daidzein. Cell proliferation inhibition is usually associated with cell cycle arresting at a specific stage. To examine if daidzein causes cell cycle arrest, we performed fluorescence-activated cell sorting (FACS) analysis. In the asynchronized steady state, the percentage of proportion of G1 phase cells was greater after treated with daidzein. In JAR cells, G1 phase 

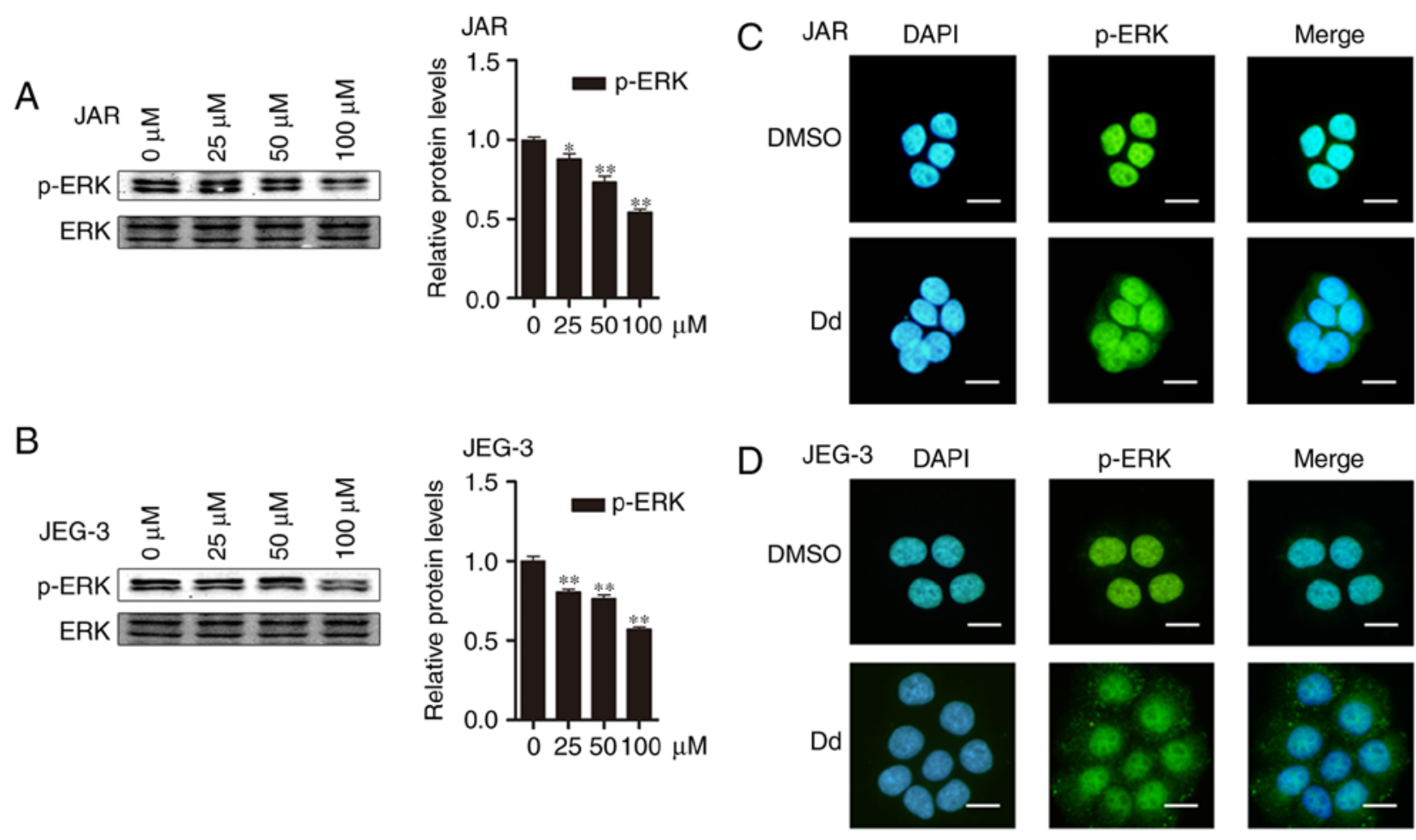

Figure 3. Changes of ERK during daidzein inhibition of choriocarcinoma cell proliferation. (A) Relative expression of p-ERK in JAR determined by western blotting. ERK was used as internal control. (B) Relative expression of p-ERK in JAR determined by western blotting. (C) The intracellular expression and distribution of p-ERK in JAR imaged by IF staining. (D) The intracellular expression and distribution of p-ERK in JEG-3. Dd stands for daidzein. Bar, $20 \mu \mathrm{m}$.

percentage of proportion was $61.66,65.38$ and $72.64 \%$ treated with 25,50 and $100 \mu \mathrm{M}$, respectively, while it was only 48.02 without (Fig. 2C). As to JEG-3 cells, daidzein increased G1 phase percentage from $50.85 \%(0 \mu \mathrm{M})$ to $56.34,60.00$ and $61.07 \%$ (25, 50 and $100 \mu \mathrm{M}$, respectively, Fig. 2D). Then we detected the expression of G1 phase related proteins (c-myc, PCNA and cyclin D1) and p21, one of CDKIs family, by western blot analysis. The expression levels of c-myc, PCNA and cyclin D1 were decreased significantly, and the decrease was greater with the increase of concentration $(\mathrm{p}<0.05$, respectively). On the contrary, the expression level of p21 increased, and the differences were also significant ( $\mathrm{p}<0.05$, respectively, Fig. 2E and F). These results suggest that choriocarcinoma cells are arrested at the G1 phase by daidzein.

Daidzein suppresses ERK1/2 phosphorylation and p-ERK1/2 nuclear translocation. When detecting expression of cell cycle associated protein, we also found that daidzein suppressed ERK1/2 by reducing phosphorylation, which suggested ERK1/2 might participate in daidzein proliferation inhibition capacity ( $\mathrm{p}<0.05$, respectively, Fig. 3A and B). As known, ERK1/2 is involved in phosphorylation and subsequent nuclear translocation, so we wondered whether daidzein might also effect nuclear translocation of ERK1/2. Therefore, we undertook to examine whether daidzein influenced this process by using immunofluorescence staining (IF), and the results showed that compared with DMSO, phospho-ERK1/2 translocated into the nucleus less after daidzein treatment (Fig. 3C and D) in both cell-lines. Taken together, our results suggested daidzein could inhibit ERK1/2 phosphorylation and p-ERK1/2 nuclear translocation.
Daidzein-caused proliferation inhibition was abolished by $C$-C6. The changes of phosphorylation and translocation of ERK1/2 were obtained during daidzein's proliferation inhibition as described above, thus we hypothesized ERK1/2 involved in daidzein-caused choriocarcinoma cell proliferation suppression. Ceramide C6 is ERK pathway agonist, and could significantly increase phosphorylation of ERK which was inhibited by daidzein in both cell lines $(\mathrm{p}<0.05$, respectively, Fig. 4A and B). At the same time, colony formation assay showed that C-C6 obviously increased the amount of cell colonies which were decreased by daidzein (Fig. 4C and D). Compared to daidzein only, C-C6 decreased G1 phase percentage from 74.56 to 63.02 and 60.75 to 54.02 in JAR and JEG-3, respectively on the basis of daidzein (Fig. 4E and F). Besides, C-C6 also abolished daidzein's increase on protein expressions of c-myc, PCNA and cyclin D1 and decreased p21 (Fig. 4G and H). In summary, daidzein's proliferation inhibition on choriocarcinoma cells can be abolished by ERK pathway agonist C-C6, therefore, we consider that ERK participated in this process.

Daidzein inhibits choriocarcinoma growth in vivo. To determine whether daidzein's anti-proliferation activity in vitro could be extended in vivo, subcutaneous xenograft model was introduced in this study. Average weights of tumors in three groups showed negative correlation with daidzein concentration (Fig. 5A). The low dose group was lighter than that of control group, and weight in high dose group was even lighter than low dose group (p<0.05, Fig. 5B). We then stained c-myc, PCNA and p-ERK expression in these JEG-3 xenograft tissues using IHC assays (Fig. 5C). The 


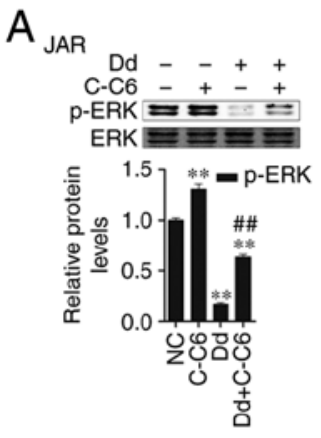

B

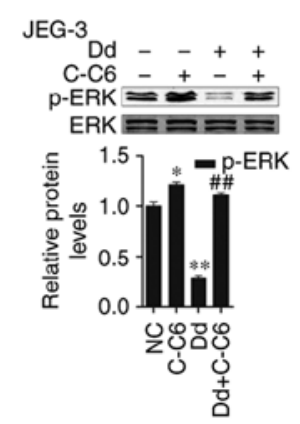

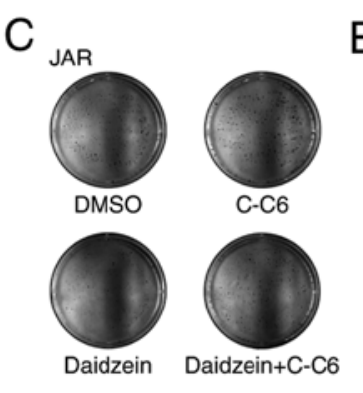

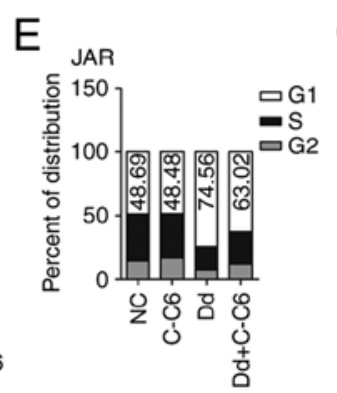

$\mathrm{D}$
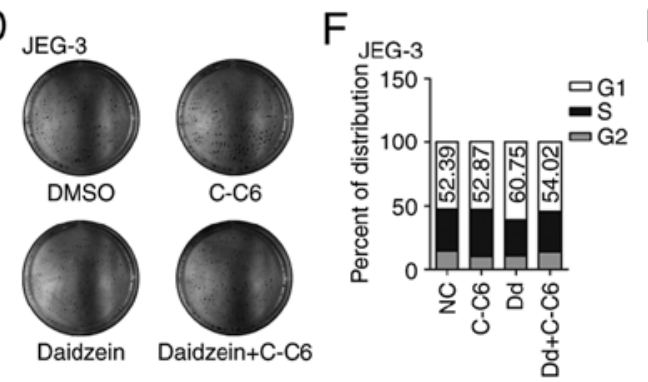
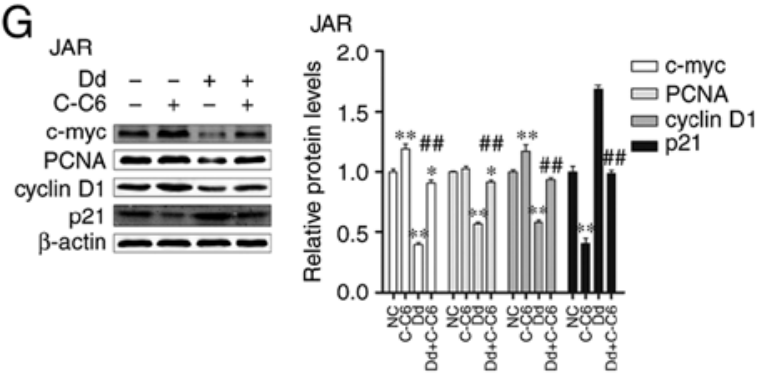

$\mathrm{H}$

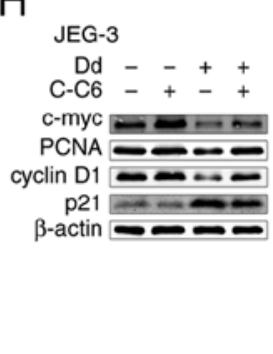

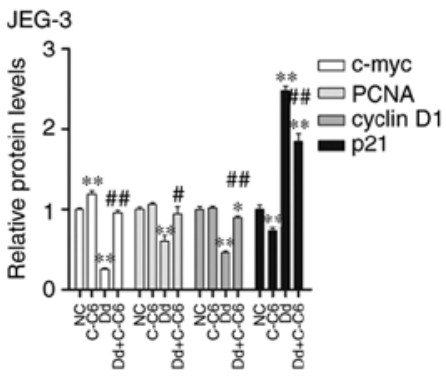

Figure 4. The influence of ERK agonist C-C6 on proliferation inhibiting ability of daidzein. (A and B) Change of p-ERK expression by C-C6 in presentation/ absence of daidzein in JAR and JEG-3. (C and D) Colony formation of JAR and JEG-3 treated by C-C6 with/without daidzein. (E and F) Cell cycle distribution of JAR and JEG-3 treated by C-C6 combined with daidzein or not. (G and H) Western blotting and the quantification of proliferation associating markers in appearance of daidzein and C-C6, single or combined. Dd stands for daidzein. Compared with $0 \mu \mathrm{M},{ }^{*} \mathrm{p}<0.05$, ${ }^{* *} \mathrm{p}<0.01$. Daidzein combined C-C6 compared to daidzein only, ${ }^{\#} \mathrm{p}<0.05,{ }^{\# \#} \mathrm{p}<0.01$.

A

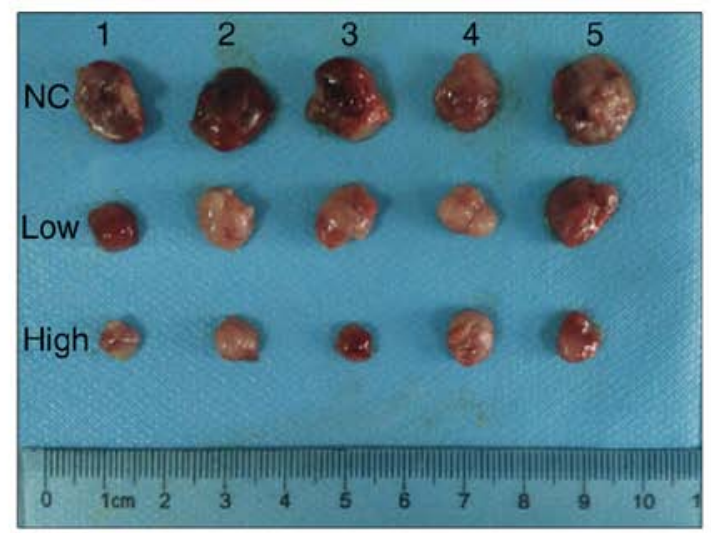

C

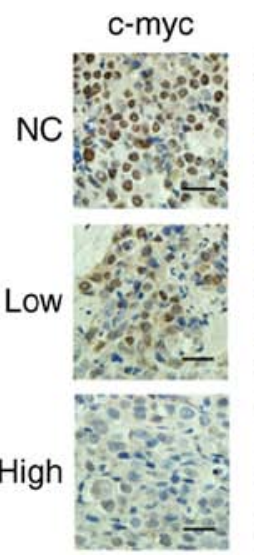

PNCA
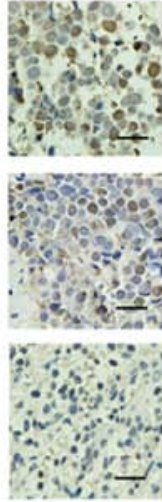

p-ERK

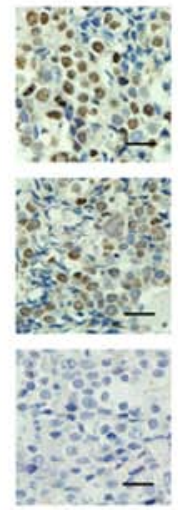

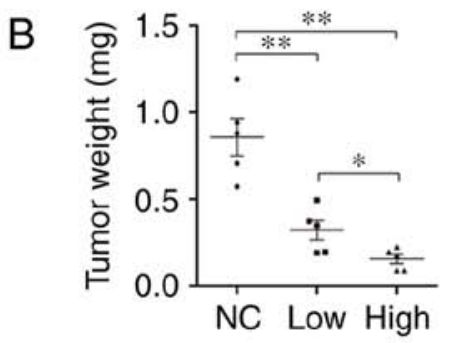
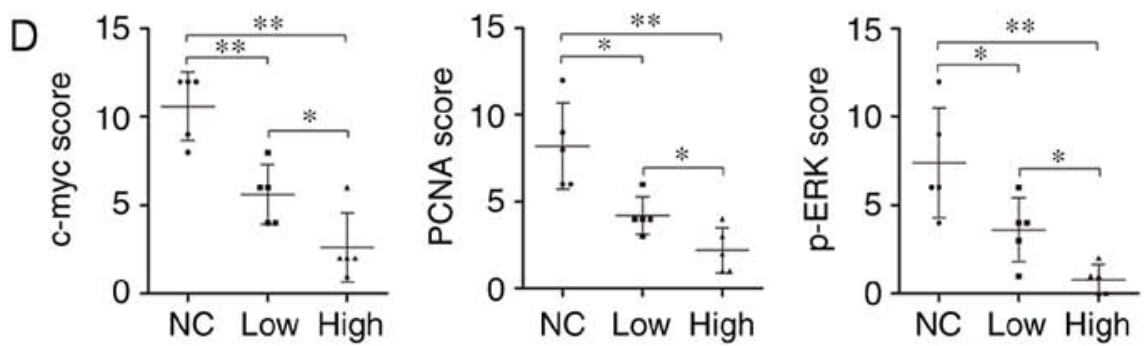

Figure 5. Daidzein effect on choriocarcinoma growth in vivo. (A) Subcutaneous xenografts of JEG-3 three weeks after subcutaneous injection (4 weeks after daily gavage with/without different concentration of daidzein). (B) Tumor weight of three groups. (C) Immunohistochemistry of tumor sections stained with c-myc, PCNA and p-ERK. (D) The IHC scores of these markers among three groups. Bar, $50 \mu \mathrm{m}$. Compared with NC group, ${ }^{*} \mathrm{p}<0.05,{ }^{* *} \mathrm{p}<0.01$.

reduced c-myc and PCNA expression was accompanied with less p-ERK staining in low dose group and even less in high dose group than control. The IHC scores of c-myc, PCNA and p-ERK in control group were $10.60 \pm 1.95,8.20 \pm 2.49$ and $7.40 \pm 3.13$, those in low dose group were $5.60 \pm 1.67,4.20 \pm 1.10$ and $3.60 \pm 1.82$, while those in high dose group were $2.60 \pm 1.95$, 
$2.20 \pm 1.30$ and $0.80 \pm 0.84$, respectively, with significant differences, and the scores of c-myc and PCNA showed positive correlation with p-ERK (Fig. 5D). These data suggested that daidzein could also inhibit choriocarcinoma growth in vivo by downregulating c-myc and PCNA, and this process was probably mediated by $\mathrm{p}-\mathrm{ERK}$.

\section{Discussion}

Choriocarcinoma is a highly malignant tumor arising from abnormal gestational trophoblasts proliferation. The use of chemotherapy has dramatically improved the prognosis of these lesions. However, despite serious side effect of chemotherapy, there are still some patients who become drug-resistant or relapse, and may finally die of liver or brain metastasis (30). Thus, it is meaningful to investigate inhibition of choriocarcinoma cell proliferation without increasing the patients side-effect burden.

Daidzein is a member of isoflavones, and is easily gained from food especially soybeans or soy-based product (31-33). Daidzein has been studied in many tumors due to its anti-proliferation action $(16,21,24)$. In choriocarcinoma, daidzein shows its regulatory function in hCG production (25). In this study, we performed in vitro and in vivo experiments to examine the effects of daidzein on proliferation of choriocarcinoma and explore the underlying mechanism. Also, our results demonstrate an antiproliferation action of daidzein on choriocarcinoma.

Daidzein has shown its antiproliferation function in various cells by cell cycle arrest. Daidzein can induce accumulation of cells in G1 phase in human melanoma cells (34), and cause cell cycle arrest at $\mathrm{G} 1$ and $\mathrm{G} 2 / \mathrm{M}$ phase in human breast cancer MCF-7 and MDA-MB-453 cells (19). Daidzein can also block G1 phase cell cycle progression of Swiss 3T3, an immortal line of fibroblast-like cells (35). In this study, we found that daidzein inhibited choriocarcinoma cell-lines JAR and JEG-3 growth rate and clone formation in a time- and concentration-dependent manner, during which cell cycle was arrested at $\mathrm{G} 1$ phase in both cell types detected by flow cytometer. At the same time, the expression of cyclin D1 was reduced by daidzein, as well as c-myc and PCNA, which can also help cell cycle progression. While the expression of p21, one of CKDIs, increased. These results strongly support that daidzein can inhibit proliferation by cell cycle arrest at G1 phase.

ERK1/2 is one of MAPK family members involved in proliferation and other cell activities (36-38). Daidzein has been reported to suppress ERK1/2 activation during its antiproliferation function in tumors $(26,27)$. In this study, during daidzein treatment, the expression of phospho-ERK1/2 was reduced, determined by western blotting. Immunofluorescence staining showed that p-ERK translocation into the nucleus was obstructed as more p-ERK stayed in the cytoplasm suggesting that daidzein suppress ERK activation by inhibiting phosphorylation and translocation into the nucleus. Then we used ERK agonist C-C6 to confirm whether ERK was involved in daidzein proliferation in JAR and JEG-3. Unsurprisingly, compared with daidzein treatment only, C-C6 increased colony formation of both cells, and also decreased G1 phase percentage. At the same time, cyclin D1, c-myc and PCNA reduction and p21 increase were weakened or offset. Collectively, daidzein inhibits choriocarcinoma cells proliferation by cell cycle arrest at G1 phase via ERK pathway in vitro.

Furthermore, we performed experiments in vivo to see if this proliferation inhibition could be still effective in vivo. It has been reported that daidzein and its metabolite can cause tumor growth inhibition in other tumors in vivo (39-41). Similar effect was also obtained in our study, as daidzein also inhibited subcutaneous xenograft growth. Average tumor weight in control group was much higher than that of low dose group, while weight of high dose group was even lower. Then IHC scores of c-myc, PCNA and p-ERK in these tumor tissue slides showed similar tendency with western blotting in vitro experiment: daidzein addition decrease p-ERK expression in xenografts as well as c-myc and PCNA, and the higher dose we gave, the more effect we gained.

In conclusion, we demonstrated that daidzein can inhibit choriocarcinoma cell proliferation in vitro and in vivo, and underlying mechanism behind the inhibitory effects may probably be suppressing ERK pathway and afterwards arresting cell cycle at G1 phase. Our findings provide new insight into the application of daidzein in treatment of choriocarcinoma to improve therapy efficiency.

\section{Acknowledgements}

This study was supported by the National Natural Science Foundation of China (no. 81172489).

\section{References}

1. Bolze PA, Attia J, Massardier J, Seckl MJ, Massuger L, van Trommel N, Niemann I, Hajri T, Schott AM and Golfier F; EOTTD group: Formalised consensus of the European Organisation for Treatment of Trophoblastic Diseases on management of gestational trophoblastic diseases. Eur J Cancer 51: 1725-1731, 2015.

2. Ryu N, Ogawa M, Matsui H, Usui H and Shozu M: The clinical characteristics and early detection of postpartum choriocarcinoma. Int J Gynecol Cancer 25: 926-930, 2015.

3. Ngu SF and Chan KK: Management of chemoresistant and quiescent gestational trophoblastic disease. Curr Obstet Gynecol Rep 3: 84-90, 2014.

4. Alazzam M, Tidy J, Osborne R, Coleman R, Hancock BW and Lawrie TA: Chemotherapy for resistant or recurrent gestational trophoblastic neoplasia. Cochrane Database Syst Rev 1: CD008891, 2016.

5. Rumer KK, Post MD, Larivee RS, Zink M, Uyenishi J, Kramer A, Teoh D, Bogart K and Winn VD: Siglec-6 is expressed in gestational trophoblastic disease and affects proliferation, apoptosis and invasion. Endocr Relat Cancer 19: 827-840, 2012.

6. Siu MK, Yeung MC, Zhang H, Kong DS, Ho JW, Ngan HY, Chan DC and Cheung AN: p21-activated kinase-1 promotes aggressive phenotype, cell proliferation, and invasion in gestational trophoblastic disease. Am J Pathol 176: 3015-3022, 2010.

7. Liu J, Shen M, Yue Z, Yang Z, Wang M, Li C, Xin C, Wang Y, Mei Q and Wang Z: Triptolide inhibits colon-rectal cancer cells proliferation by induction of G1 phase arrest through upregulation of p21. Phytomedicine 19: 756-762, 2012.

8. Ju SM, Lee J, Kang JG, Jeong SO, Park JH, Pae HO, Lee GS Kim WS, Lyu YS and Jeon BH: Nardostachys chinensis induces granulocytic differentiation with the suppression of cell growth through p27 (Kip1) protein-related G0/G1 phase arrest in human promyelocytic leukemic cells. Pharm Biol 53: 1002-1009, 2015.

9. Gehen SC, Vitiello PF, Bambara RA, Keng PC and O'Reilly MA: Downregulation of PCNA potentiates p21-mediated growth inhibition in response to hyperoxia. Am J Physiol Lung Cell Mol Physiol 292: L716-L724, 2007. 
10. Faouzi M, Kischel P, Hague F, Ahidouch A, Benzerdjeb N, Sevestre H, Penner R and Ouadid-Ahidouch H: ORAI3 silencing alters cell proliferation and cell cycle progression via c-myc pathway in breast cancer cells. Biochim Biophys Acta 1833: 752-760, 2013

11. Zhang F, Kong DS, Zhang ZL, Lei N, Zhu XJ, Zhang XP, Chen L, Lu Y and Zheng SZ: Tetramethylpyrazine induces G0/G1 cell cycle arrest and stimulates mitochondrial-mediated and caspasedependent apoptosis through modulating ERK/p53 signaling in hepatic stellate cells in vitro. Apoptosis 18: 135-149, 2013.

12. Liu E, Li J, Shi S, Wang X, Liang T, Wu B and Li Q: Sustained ERK activation-mediated proliferation inhibition of farrerol on human gastric carcinoma cell line by G0/G1-phase cell-cycle arrest. Eur J Cancer Prev 25: 490-499, 2016.

13. Zhao HB, Tang CL, Hou YL, Xue LR, Li MQ, Du MR and Li DJ: CXCL12/CXCR4 axis triggers the activation of EGF receptor and ERK signaling pathway in CsA-induced proliferation of human trophoblast cells. PLoS One 7: e38375, 2012.

14. Xie Y, Cui D, Sui L, Xu Y, Zhang N, Ma Y, Li Y and Kong Y: Induction of forkhead box M1 (FoxM1) by EGF through ERK signaling pathway promotes trophoblast cell invasion. Cell Tissue Res 362: 421-430, 2015.

15. Liggins J, Mulligan A, Runswick S and Bingham SA: Daidzein and genistein content of cereals. Eur J Clin Nutr 56: 961-966, 2002 .

16. Hsu A, Bray TM, Helferich WG, Doerge DR and Ho E: Differential effects of whole soy extract and soy isoflavones on apoptosis in prostate cancer cells. Exp Biol Med (Maywood) 235: 90-97, 2010.

17. Lu Z, Zhou R, Kong Y, Wang J, Xia W, Guo J, Liu J, Sun H, Liu K, Yang J, et al: S-equol, a secondary metabolite of natural anticancer isoflavone daidzein, inhibits prostate cancer growth in vitro and in vivo, Though activating the Akt/FOXO3a pathway. Curr Cancer Drug Targets 16: 455-465, 2016.

18. Charalambous C, Pitta CA and Constantinou AI: Equol enhances tamoxifen's anti-tumor activity by induction of caspase-mediated apoptosis in MCF-7 breast cancer cells. BMC Cancer 13: 238 , 2013.

19. Choi EJ and Kim GH: Daidzein causes cell cycle arrest at the G1 and G2/M phases in human breast cancer MCF-7 and MDA-MB-453 cells. Phytomedicine 15: 683-690, 2008.

20. Guo JM, Xiao BX, Liu DH, Grant M, Zhang S, Lai YF, Guo YB and Liu Q: Biphasic effect of daidzein on cell growth of human colon cancer cells. Food Chem Toxicol 42: 1641-1646, 2004.

21. Iwashita K, Kobori M, Yamaki K and Tsushida T: Flavonoids inhibit cell growth and induce apoptosis in B16 melanoma 4A5 cells. Biosci Biotechnol Biochem 64: 1813-1820, 2000.

22. Park HJ, Jeon YK, You DH and Nam MJ: Daidzein causes cytochrome $c$-mediated apoptosis via the Bcl-2 family in human hepatic cancer cells. Food Chem Toxicol 60: 542-549, 2013.

23. Tang S, Hu J, Meng Q, Dong X, Wang K, Qi Y, Chu C, Zhang X and Hou L: Daidzein induced apoptosis via down-regulation of $\mathrm{Bcl}-2 / \mathrm{Bax}$ and triggering of the mitochondrial pathway in BGC-823 cells. Cell Biochem Biophys 65: 197-202, 2013.

24. Guo JM, Kang GZ, Xiao BX, Liu DH and Zhang S: Effect of daidzein on cell growth, cell cycle, and telomerase activity of human cervical cancer in vitro. Int J Gynecol Cancer 14 882-888, 2004

25. Jeschke U, Briese V, Richter DU, Bruer G, Plessow D, Waldschläger J, Mylonas I and Friese K: Effects of phytoestrogens genistein and daidzein on production of human chorionic gonadotropin in term trophoblast cells in vitro. Gynecolo Endocrinol 21: 180-184, 2005.

26. Bielecki A, Roberts J, Mehta R and Raju J: Estrogen receptor- $\beta$ mediates the inhibition of DLD-1 human colon adenocarcinoma cells by soy isoflavones. Nutr Cancer 63: 139-150, 2011.
27. He Y, Wu X, Cao Y, Hou Y, Chen H, Wu L, Lu L, Zhu W and $\mathrm{Gu}$ Y: Daidzein exerts anti-tumor activity against bladder cancer cells via inhibition of FGFR3 pathway. Neoplasma 63: 523-531, 2016.

28. Lim TG, Kim JE, Lee SY, Park JS, Yeom MH, Chen H, Bode AM, Dong Z and Lee KW: The daidzein metabolite, $6,7,4$-Trihydroxyisoflavone, is a novel inhibitor of PKC $\alpha$ in suppressing solar UV-induced matrix metalloproteinase 1 . Int J Mol Sci 15: 21419-21432, 2014.

29. Kang NJ, Lee KW, Rogozin EA, Cho YY, Heo YS, Bode AM, Lee HJ and Dong Z: Equol, a metabolite of the soybean isoflavone daidzein, inhibits neoplastic cell transformation by targeting the MEK/ERK/p90RSK/activator protein-1 pathway. J Biol Chem 282: 32856-32866, 2007.

30. Seckl MJ, Sebire NJ and Berkowitz RS: Gestational trophoblastic disease. Lancet 376: 717-729, 2010

31. Bao C, Namgung H, Lee J, Park HC, Ko J, Moon H, Ko HW and Lee HJ: Daidzein suppresses tumor necrosis factor- $\alpha$ induced migration and invasion by inhibiting hedgehog/Glil signaling in human breast cancer cells. J Agric Food Chem 62: 3759-3767, 2014.

32. Choi EJ and Kim GH: Antiproliferative activity of daidzein and genistein may be related to ER $\alpha / \mathrm{c}-$ erbB-2 expression in human breast cancer cells. Mol Med Rep 7: 781-784, 2013.

33. Liu X, Suzuki N, Santosh Laxmi YR, Okamoto Y and Shibutani S: Anti-breast cancer potential of daidzein in rodents. Life Sci 91: 415-419, 2012.

34. Casagrande F and Darbon JM: Effects of structurally related flavonoids on cell cycle progression of human melanoma cells: Regulation of cyclin-dependent kinases CDK2 and CDK1. Biochem Pharmacol 61: 1205-1215, 2001.

35. Higashi K and Ogawara H: Daidzein inhibits insulin- or insulinlike growth factor-1-mediated signaling in cell cycle progression of Swiss 3T3 cells. Biochim Biophys Acta 1221: 29-35, 1994.

36. Ito T, Yamada S, Tanaka C, Ito S, Murai T, Kobayashi D, Fujii T, Nakayama G, Sugimoto H, Koike M, et al: Overexpression of L1CAM is associated with tumor progression and prognosis via ERK signaling in gastric cancer. Ann Surg Oncol 21: 560-568, 2014.

37. Sadaria MR, Yu JA, Meng X, Fullerton DA, Reece TB and Weyant MJ: Secretory phospholipase A2 mediates human esophageal adenocarcinoma cell growth and proliferation via ERK 1/2 pathway. Anticancer Res 33: 1337-1342, 2013.

38. Han S, Li Z, Master LM, Master ZW and Wu A: Exogenous IGFBP-2 promotes proliferation, invasion, and chemoresistance to temozolomide in glioma cells via the integrin $\beta 1$-ERK pathway. Br J Cancer 111: 1400-1409, 2014.

39. Singh-Gupta V, Zhang H, Yunker CK, Ahmad Z, Zwier D, Sarkar FH and Hillman GG: Daidzein effect on hormone refractory prostate cancer in vitro and in vivo compared to genistein and soy extract: Potentiation of radiotherapy. Pharm Res 27: 1115-1127, 2010.

40. Lee DE, Lee KW, Jung SK, Lee EJ, Hwang JA, Lim TG, Kim BY, Bode AM, Lee HJ and Dong Z: 6,7,4'-trihydroxyisoflavone inhibits HCT-116 human colon cancer cell proliferation by targeting CDK1 and CDK2. Carcinogenesis 32: 629-635, 2011.

41. Somjen D, Grafi-Cohen M, Katzburg S, Weisinger G, Izkhakov E, Nevo N, Sharon O, Kraiem Z, Kohen F and Stern N: Anti-thyroid cancer properties of a novel isoflavone derivative, 7-(O)-carboxymethyl daidzein conjugated to N-t-Bochexylenediamine in vitro and in vivo. J Steroid Biochem Mol Biol 126: 95-103, 2011. 